\title{
Effects of surface electrical stimulation during sitting on pelvic floor muscle function and sexual function in women with stress urinary incontinence
}

\author{
Ui-Jae Hwang, PhD, $\mathrm{PT}^{1}$, Oh-Yun Kwon, PhD, $\mathrm{PT}^{2}$, Min-Seok Lee, MD \\ Department of Physical Therapy, ${ }^{1}$ Graduate School, ${ }^{2}$ College of Health Science, Laboratory of Kinetic Ergocise Based on Movement Analysis, Yonsei \\ University, Wonju; ${ }^{3}$ Sophie-Marceau Women's Clinic, Daegu, Korea
}

\section{Objective}

Dysfunction of the pelvic floor muscles (PFM) is associated with sexual dysfunction in women with stress urinary incontinence (SUI). The EasyK7 device was developed to stimulate the PFM by surface electrical stimulation during sitting (SESdS). We investigated the effects of SESdS on PFM function and sexual function in women with SUI.

\section{Methods}

Women with SUI were randomized into the SESdS and control groups. PFM function and sexual function were assessed using a perineometer and the pelvic organ prolapse-urinary incontinence sexual function questionnaire (PISQ), respectively. After 8 weeks, the groups were compared using either analysis of covariance with the baseline values as covariates or the paired Student's $t$-test.

\section{Results}

The final analysis included 16 subjects from each group. There were significant differences between the SESdS and control groups after the intervention, as well as within the SESdS group between the pre- and post-intervention measurements. The $P$-values for the differences in PFM measurements between the groups, and between the preand post- intervention measurements within the SESdS group, were 0.001 and 0.004 for power, 0.015 and 0.011 for strength, and 0.012 and 0.034 for endurance, respectively. In addition, in the PISQ, there were significant differences between the groups and between the pre- and post-intervention measurements within the SESdS group in the partner-related domain (between groups: $P=0.003$; within SESdS group: $P=0.024$ ) and total score (between groups: $P<0.001$; within SESdS group: $P=0.001$ ).

\section{Conclusion}

SESdS can improve PFM function and sexual function in women with SUI.

\section{Trial Registrati}

Clinical Research Information Service Identifier: КСT0003357

Keywords: Electrical stimulation; Pelvic floor; Sexual dysfunctions; Stress urinary incontinence

\section{Introduction}

Female sexual dysfunction (FSD) is highly prevalent among women with pelvic floor dysfunction, such as those with stress urinary incontinence (SUI). SUI may have a negative effect on female sexual function [1-3]. The potential causes of FSD include the fear that incontinence may produce an embarrassing odor during penetration or intercourse [4,5]. A previous study suggested that women with SUI experienced
Received: 2019.08.21. Revised: 2019.10.21. Accepted: 2019.11.06. Corresponding author: Min-Seok Lee, MD

Sophie-Marceau Women's Clinic, 2395 Dalgubeol-daero, Suseonggu, Daegu 42019, Korea

E-mail: msleesena@hanmail.net

https://orcid.org/0000-0002-0028-8767

Articles published in Obstet Gynecol Sci are open-access, distributed under the terms of the Creative Commons Attribution Non-Commercial License (http://creativecommons. org/licenses/by-nc/3.0/) which permits unrestricted non-commercial use, distribution, and reproduction in any medium, provided the original work is properly cited.

Copyright $\odot 2020$ Korean Society of Obstetrics and Gynecology 


\section{Obstetrics \& Gynecology Science}

Ui-Jae Hwang, et al. SESdS on PFM and sexual function

lower sexual satisfaction than those without urinary disorders [3].

SUI is caused by weak or damaged pelvic floor muscles (PFM) and connective tissues, that support the urethra. Proper functioning of the PFM is important for a woman's pleasure during vaginal intercourse and for the strength of grip felt by her partner [6]. During orgasm, involuntary rhythmic contractions are generated by the PFM, including the iliococcygeus and pubococcygeus muscles.

The aim of non-invasive treatment for FSD in women with $\mathrm{SUI}$ is to enhance the function of the PFM $[7,8]$. PFM training (PFMT) using electrical stimulation (ES) may reduce urine leakage and improve female sexual function, as well as the strength and force of PFM contraction. Previous studies have shown that intravaginal ES, used as part of a rehabilitation program to treat SUI, may also be used to treat FSD [8-10]. Women with dysfunctional PFM showed improved sexual function after PFMT $[8,11]$. However, although various characteristics of the PFM (strength, power, and endurance) are functionally important, previous studies have focused only on the effect of ES on PFM strength $[12,13]$.

The EasyK7 device (Alphamedic Co., Ltd., Daegu, Korea) was recently developed to improve the function of the PFM by surface ES during sitting (SESdS) via transcutaneous electrodes in contact with both the perivaginal and sacral regions. PFMT using ES is usually administered via a transvaginal or transanal electrode. This procedure is invasive, dependent on the degree of vaginal space, associated with hygiene problems and discomfort, leading to low levels of adherence [14]. Correia et al. [15] demonstrated that both surface ES (SES) and intravaginal ES can improve quality of life by enhancing the strength and force of contraction of the PFM. Green and Laycock [16] found that patients regarded intravaginal ES as too invasive. In contrast, the application of SES is more comfortable and potentially more acceptable to women with SUI [17].

Previous studies have investigated the effects of SES on patients in a supine or hook-lying position. This treatment is usually administered via transcutaneous electrodes in contact with the perivaginal or sacral region [15,18-20]. However, no study has investigated improvements in PFM function (muscle strength, power, and endurance) after SESdS. Therefore, the present study investigated the effects of SESdS on PFM function and sexual function in women.

\section{Materials and methods}

\section{Subjects and design}

The present study was performed between September 2018 and December 2018 at an obstetrics and gynecology clinic in Seoul, South Korea. An investigator-blinded, parallelgroup, randomized controlled trial was performed, including both control and SESdS groups (1:1). Based on pilot data gathered from 3 subjects in each group, $G *$ Power software (ver. 3.1.3; University of Trier, Trier, Germany) [21] was used to calculate the sample size needed to achieve a power of 0.80 and an effect size of 0.917 with an a level of 0.05 . The sample size calculation indicated that 6 more subjects must be recruited to each group. Subjects were recruited using advertisements that provided telephone contact details to women with SUI who may be interested in participating in the study. Visits were scheduled to determine the applicants' suitability for the study, based on appropriate inclusion and

Table 1. Inclusion and exclusion criteria

\begin{tabular}{ll}
\hline Criteria for the study & Characteristics \\
\hline Inclusion criteria & SUl diagnosed by a urogynecologist \\
Leakage episode occurring more than & once per week \\
Body mass index $<30 \mathrm{~kg} / \mathrm{m}^{2}$ \\
Age between 30 and 60 years \\
Non-smoker \\
Not addicted to alcohol or drugs \\
Successfully completed the medical \\
screening questionnaire \\
Not fulfilling the inclusion criteria \\
Urogenital prolapse grade III or higher \\
Cardiac pacemaker \\
Device implanted in the pelvis or hip \\
joint \\
Pregnant/planning to get pregnant \\
Pelvic or abdominal surgery within the \\
last 6 months \\
Aversion to SESdS \\
Concomitant treatment for SUl during \\
the trial period \\
Neurological or psychiatric disease \\
Urinary tract infection \\
\hline
\end{tabular}

SUI, stress urinary incontinence; SESdS, surface electrical stimulation during sitting. 


\section{Obstetrics \& Gynecology Science}

Vol. 63, No. 3, 2020

exclusion criteria. The severities of incontinence and FSD were determined in interviews.

The inclusion/exclusion criteria for the study are shown in Table 1. A total of 34 subjects who met these criteria were randomly separated into 2 groups (www.randomization. com): a control and an SESdS group (Fig. 1).

\section{Surface electrical stimulation during sitting}

The EasyK7 is a SESdS device that stimulates the PFM and surrounding structures using 3 surface electrodes in contact with the perivaginal and sacral regions. In the present study, the surface electrodes were positioned near each participant's anus and sacrum to stimulate both the perivaginal and sacral regions, with the subject sitting on the EasyK7 device (Fig. 2). Subjects were asked to sit on the device to ensure that both electrodes made contact with the perivaginal and sacral regions. The amplitude used for stimulation was set to a comfortable level for each subject. The Easyk7 deliv- ered biphasic and asymmetric impulses of $25 \mathrm{~Hz}$ at pulses of 11 seconds, with an 11-second rest period between pulses. The mean intensities used were 19.37 $\pm 6.29 \mathrm{~mA}$ (range, 2.5-30 mA). Each EasyK7 session was 15 minutes long.

\section{Intervention}

The subjects in the SESdS group were provided with an EasyK7 device and shown how to use and maintain the device correctly. These subjects were instructed to use the device for a single 15-minute session per day for 5-6 days per week, for a total of 8 weeks. In addition, the subjects were permitted to increase the EasyK 7 stimulation amplitude within tolerable limits.

Control group subjects walked for more than 20 minutes in lieu of Easyk7 treatments. At the end of the 8-week intervention period, we provided an EasyK 7 device as a reward to all subjects for participating in the study. Measurements were recorded in both groups before and after the 8-week inter-

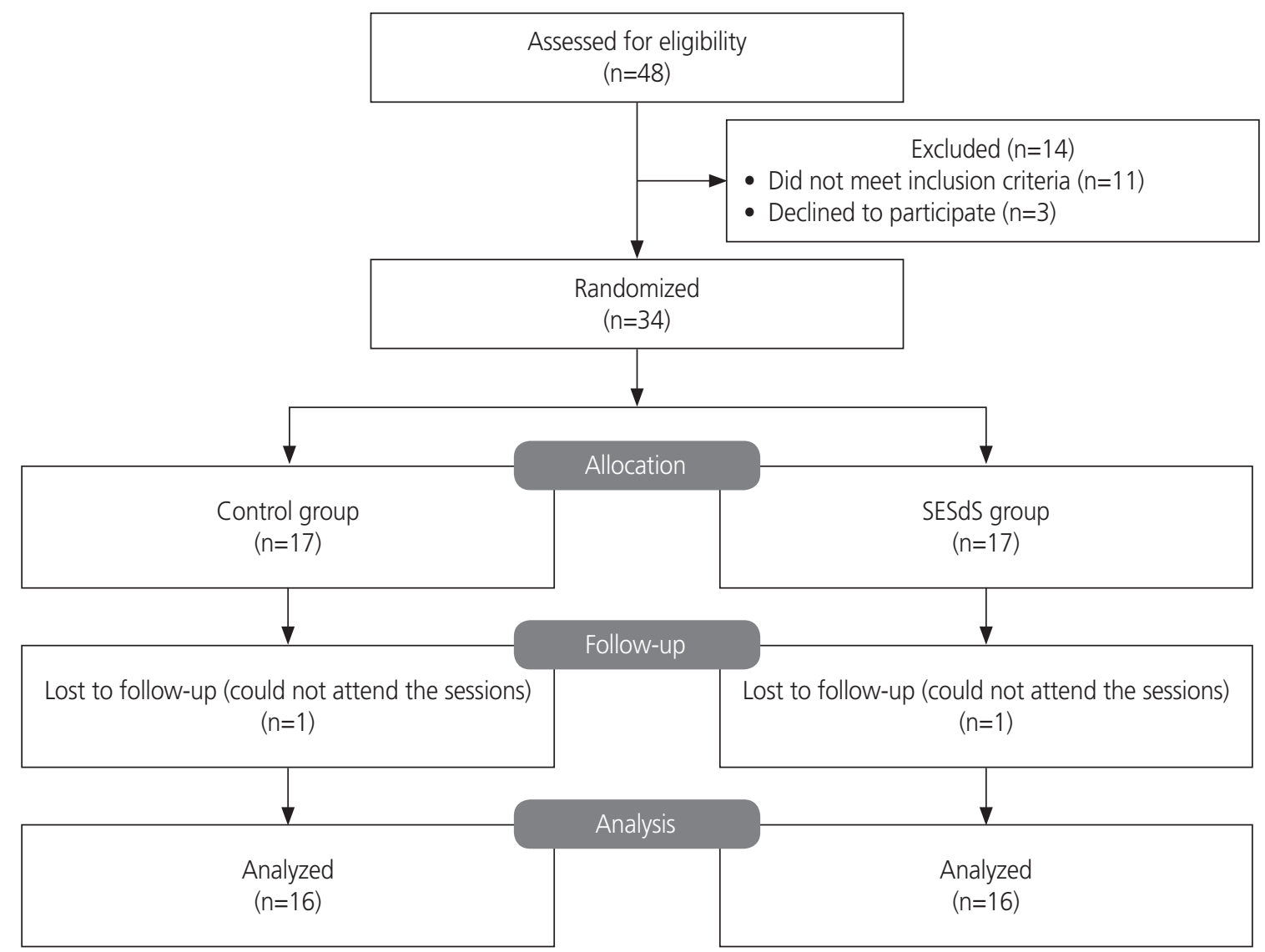

Fig. 1. Flow chart of patient selection into this randomized trial of transcutaneous electrical stimulation in women with stress urinary incontinence. SESdS, surface electrical stimulation during sitting. 


\section{Obstetrics \& Gynecology Science}

Ui-Jae Hwang, et al. SESdS on PFM and sexual function

vention period. We measured PFM function using a perineometer and evaluated sexual function using a questionnaire.

\section{Outcomes}

Female sexual function was measured using the Korean version of the pelvic organ prolapse-urinary incontinence sexual function questionnaire (PISQ) [22]. The PISQ is a 31-item questionnaire with the responses based on a 5-point Likert scale [23]. It has been used to evaluate sexual function in women with urinary incontinence and/or pelvic organ prolapse [24]. The PISQ has 3 distinct domains that cover behavioral/emotive (15 items), physical (10 items), and partner-related (6 items) aspects of female sexual function. There is also an overall total score. The behavioral/emotive domain assesses sexual desire, frequency of sexual activity, and orgasm capability. The physical domain evaluates the effect of urinary incontinence on sexual function. The partnerrelated domain evaluates the participant's perception of her partner's response to the effect of pelvic floor disorders on the couple's sex life. Each domain score is calculated by adding the scores from the individual items in each domain. The total PISQ-31, physical domain, behavioral/emotive domain, and partner-related domain scores range from 0 to 125,0 to 40,0 to 61 , and 0 to 24 , respectively. In all domains, higher scores indicate better sexual function.

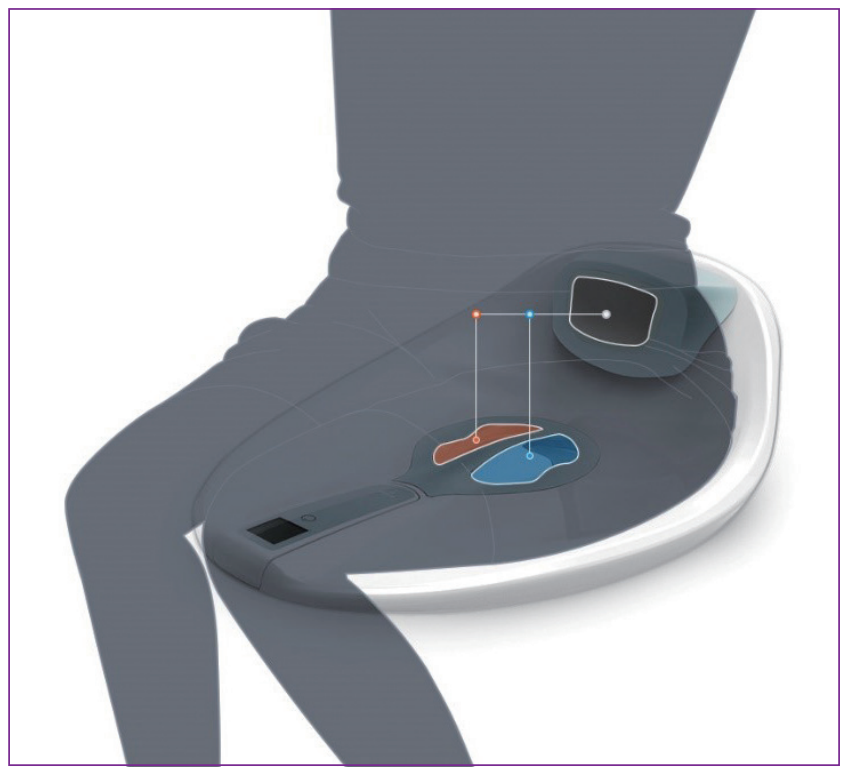

Fig. 2. Surface electrical stimulation during sitting using the Alphamedic EasyK7 device.
PFM function was evaluated using a vaginal pressure measurement device (VVP-3000 perineometer; QLMED, Ltd., Seongnam, Korea; length: $115 \mathrm{~mm}$, active surface length: $66 \mathrm{~mm}$, diameter: $24 \mathrm{~mm}$ ), with each participant in the hook-lying position. A microprocessor with latex tubing was connected to the vaginal pressure probe and transmitted pressure readings when vaginal contractions compressed the probe. The baseline pressure value when the PFM was relaxed was set to $0 \mathrm{mmHg}$. Subjects were instructed to contract their PFM at maximum effort for 3 seconds. Next, they were asked to contract their PFM in an inward direction as much as possible, without contracting their abdominal or gluteal muscles [25]. The strength of the PFM was measured in $\mathrm{mm} \mathrm{Hg}$ based on the difference between resting pressure and peak pressure. The mean value of 2 maximal voluntary contractions (MVCs) was recorded [26]. To measure muscle power, the subjects performed MVCs as rapidly as possible [27]. We defined the power of the PFM as the peak pressure divided by the time required to achieve MVC $(\mathrm{mmHg} / \mathrm{s})$. The time required to achieve MVC was measured from the starting point until peak pressure was achieved. We defined the endurance of the PFM as the mean force of vaginal contraction for 10 seconds during a single attempt.

\section{Statistical analyses}

Kolmogorov-Smirnov Z-tests were used to confirm that the data were normally distributed. Analysis of covariance was used to compare variables between groups and within each group before and after the intervention. Baseline values were used as covariates. In addition, the paired Student's t-test was used for comparisons before and after the intervention in each group. All data are expressed as mean \pm standard deviation. Effect sizes were calculated to estimate the significance of differences between groups. Each effect size ( $r$ ) ranged from 0 (no effect) to 1 (complete effect). Effect sizes of 0 to $<0.1,0.1$ to $<0.3,0.3$ to $<0.5$, and $\geq 0.5$ were categorized as no effect, small effect, moderate effect, and large effect, respectively. All statistical analyses were performed using SPSS software (ver. 18.0; SPSS Inc., Chicago, IL, USA), and a $P$-value $<0.05$ was considered statistically significant.

\section{Results}

A total of 34 subjects with SUI were randomly separated into 


\section{Obstetrics \& Gynecology Science}

Vol. 63, No. 3, 2020

2 groups, each consisting of 17 subjects. However, due to time constraints, one subject from each group failed to complete the intervention period. Therefore, 32 subjects completed the protocol and were included in the analysis (Table 2). There were no significant differences in demographic characteristics or Ingelman-Sundberg scale values between the 2 groups.

Table 3 shows the SESdS-induced improvements in PFM function and PISQ parameters, determined by comparing the SESdS and control groups after the intervention period, as well as improvements within the SESdS group (i.e., the differ- ence between measurements recorded before and after intervention). With regard to PFM function, there were significant differences between the groups, as well as between the pre- and post-intervention measurements within the SESdS group, in terms of power (between groups: $P=0.001$; within SESdS group: $P=0.004$ ), strength (between groups: $P=0.015$; within SESdS group: $P=0.011$ ), and endurance (between groups: $P=0.012$; within SESdS group: $P=0.034$ ). In addition, PFM function significantly increased after intervention within the SESdS group (strength: $P=0.019$; power: $P=0.03$; endurance: $P=0.018$ ). However, there were no significant dif-

Table 2. Characteristics of the participants

\begin{tabular}{lccc}
\hline Characteristics & Control group $(\mathbf{n = 1 6 )}$ & SESdS group $(\mathbf{n}=\mathbf{1 6})$ & $\boldsymbol{P}$-value \\
\hline Age $(\mathrm{yr})$ & $41.1 \pm 7.2$ & $42.3 \pm 9.1$ & 0.684 \\
Body mass index $\left(\mathrm{kg} / \mathrm{m}^{2}\right)$ & $22.8 \pm 3.5$ & $22.6 \pm 2.8$ & 0.825 \\
Duration of symptoms $(\mathrm{yr})$ & $7.8 \pm 6.0$ & $5.7 \pm 3.6$ & 0.246 \\
No. of deliveries & $1.5 \pm 0.9$ & $1.9 \pm 0.7$ & 0.201 \\
Vaginal deliveries & $1.5 \pm 0.9$ & $1.5 \pm 1.0$ & 1.000 \\
Ingelman-Sundberg scale & $1.4 \pm 0.6$ & $1.1 \pm 0.3$ & 0.168 \\
\hline
\end{tabular}

Data are shown as mean \pm standard deviation.

SESdS, surface electrical stimulation during sitting.

Table 3. Primary outcomes in each group pre- and post-intervention

\begin{tabular}{|c|c|c|c|c|c|c|c|}
\hline \multicolumn{3}{|c|}{ Primary outcomes } & \multirow{2}{*}{$\begin{array}{c}\text { Pre-intervention } \\
16.41 \pm 13.20\end{array}$} & \multirow{2}{*}{$\begin{array}{c}\text { Post-intervention } \\
15.16 \pm 10.07\end{array}$} & \multirow{2}{*}{$\begin{array}{c}\text { Effect size } \\
0.32\end{array}$} & \multirow{2}{*}{$\begin{array}{c}\begin{array}{c}\text { Within } \boldsymbol{P} \text { - } \\
\text { value }\end{array} \\
0.418\end{array}$} & \multirow{2}{*}{$\begin{array}{c}\begin{array}{c}\text { Between } \\
\boldsymbol{P} \text {-value }\end{array} \\
0.001\end{array}$} \\
\hline PFM & Power $(\mathrm{mmHg} / \mathrm{s})$ & Control group & & & & & \\
\hline & & SESdS group ${ }^{a)}$ & $16.27 \pm 9.20$ & $30.50 \pm 17.56$ & & 0.030 & \\
\hline & Strength $(\mathrm{mmHg})$ & Control group & $18.70 \pm 10.07$ & $19.02 \pm 9.40$ & 0.19 & 0.557 & 0.015 \\
\hline & & SESdS group ${ }^{a)}$ & $20.21 \pm 9.09$ & $26.60 \pm 11.28$ & & 0.019 & \\
\hline & Endurance (mmHg) & Control group & $13.72 \pm 7.96$ & $13.27 \pm 7.35$ & 0.20 & 0.704 & 0.012 \\
\hline & & SESdS group & $14.49 \pm 7.23$ & $20.46 \pm 10.34$ & & 0.018 & \\
\hline \multirow[t]{8}{*}{ PSIQ } & Behavioral/emotive score & Control group & $26.56 \pm 11.78$ & $23.56 \pm 10.37$ & 0.42 & 0.056 & 0.000 \\
\hline & & SESdS group ${ }^{b)}$ & $26.94 \pm 13.43$ & $33.25 \pm 15.45$ & & 0.000 & \\
\hline & Physical score & Control group & $34.81 \pm 3.29$ & $35.13 \pm 4.10$ & 0.08 & 0.714 & 0.121 \\
\hline & & SESdS group & $30.06 \pm 4.54$ & $34.56 \pm 2.97$ & & 0.000 & \\
\hline & Partner-related score & Control group & $18.25 \pm 2.08$ & $18.13 \pm 2.19$ & 0.26 & 0.697 & 0.003 \\
\hline & & SESdS group & $18.69 \pm 2.36$ & $20.13 \pm 1.71$ & & 0.022 & \\
\hline & Total score & Control group & $79.63 \pm 14.29$ & $76.81 \pm 12.10$ & 0.54 & 0.140 & 0.000 \\
\hline & & SESdS group ${ }^{\text {b) }}$ & $75.69 \pm 16.42$ & $87.69 \pm 16.76$ & & 0.000 & \\
\hline
\end{tabular}

Data are shown as mean \pm standard deviation.

PFM, pelvic floor muscles; PISQ, pelvic organ prolapse-urinary incontinence sexual function questionnaire; SESdS, surface electrical stimulation during sitting.

${ }^{\text {a) }} P<0.05 ;{ }^{\text {b) }} P<0.001$. 


\section{Obstetrics \& Gynecology Science}

Ui-Jae Hwang, et al. SESdS on PFM and sexual function

ferences in PFM function within the control group (strength: $P=0.557$; power: $P=0.418$; endurance: $P=0.704$ ).

In the PISQ, there were significant differences between the groups and within the SESdS group in the partner-related domain (between groups: $P=0.003$; within SESdS group: $P=0.024$ ) and total score (between groups: $P<0.001$; within SESdS group: $P=0.001)$. For the behavioral/emotive domain, there was a significant difference between the groups $(P<0.001)$, but not within the SESdS group $(P=0.113)$. In the physical domain, there was a significant difference within the SESdS group $(P<0.001)$, but not between the groups $(P=0.121)$. In addition, there were significant increases in all PISQ domains after intervention in the SESdS group (behavioral/emotive domain: $P<0.001$; physical domain: $P<0.001$; partner-related domain: $P=0.022$; total score: $P<0.001$ ), but there were no such increases within the control group (behavioral/emotive domain: $P=0.056$; physical domain: $P=0.714$; partner-related domain: $P=0.697$; total score: $P=0.140$ ).

\section{Discussion}

Previous studies have suggested that PFMT improves PFM and female sexual function $[12,28]$; the present study confirmed these beneficial effects after 8 weeks of SESdS. Therefore, SESdS can be used to improve sexual function in women with SUI.

We measured PFM function (strength, power, and endurance) using a perineometer. Sale and Norman defined muscle strength as the peak force during MVC and muscle power as the rate at which peak force is achieved divided by the time required to achieve MVC $[29,30]$. Muscular endurance is the muscle's capacity to maintain submaximal force $[26,31]$. In the present study, PFM function was significantly enhanced after 8 weeks of SESdS training, compared with baseline. In addition, there were significant differences in PFM function between the SESdS and control groups. Several studies that used a perineometer to measure PFM strength found that SES training can increase the force of contraction $[15,32]$. One explanation for this improved PFM function after SESdS training is that stimulating the pudendal nerve [33] may cause perturbations in myofibers and the contiguous extracellular matrix [34]. In addition, ES may randomly recruit type I (slow-twitch) and II (fast-twitch) muscle fibers [35]. The
PFM consist of 70\% slow-twitch fibers and 30\% fast-twitch fibers. Therefore, ES may enhance the strength and power of contractions by increasing the recruitment of fast-twitch muscle fibers, and it may enhance the endurance of the PFM by increasing the recruitment of slow-twitch muscle fibers. Consequently, the activation, recruitment, coordination, and proprioception of the PFM may be improved [33].

Previous studies have suggested that different modes of ES improve sexual function in women with urinary incontinence or pelvic floor disorders. Rivalta et al. [10] used a combined rehabilitation program that included ES; they reported varying degrees of improvement in sexual function in all domains measured. A study by Aydın et al. [36] demonstrated that ES can significantly improve arousal, desire, orgasm, and satisfaction. In the present study, we observed significant improvements in the partner-related domain and total PISQ score within the SESdS group (pre- vs. post-intervention) and between the groups (SESdS vs. control group). We also found significant increases in all domains of the PISQ after intervention in the SESdS group. Increases in PISQ scores after 8 weeks of SESdS may occur because stimulation of the PFM is sufficient to produce the contractions necessary for vaginal friction and blood flow $[6,12]$. Graber and Kline-Graber [6] demonstrated that the contraction strength of the pubococcygeus muscle was significantly weaker in anorgasmic women than in orgasmic women. The PFM, particularly the pubococcygeus and iliococcygeus muscles, are responsible for the involuntary rhythmic contractions that occur during orgasm, which are followed by 5-15 spasms or convulsions at 0.8-second intervals [37]. In addition, after 8 weeks of ES training, women may lose their fear of dyspareunia and urine leakage during intercourse and recover their capacity for sexual arousal and satisfaction [38]. The reasons for FSD include lack of interest, distress, and the fear that incontinence may produce an embarrassing odor during penetration or intercourse [39]. Women with SUI are more likely to experience incontinence during penetration [40]. Therefore, the improvements observed in the behavioral/emotive and physical domains of the PISQ in the present study may have occurred because incontinence during intercourse was no longer a problem, while the improvements in sexual arousal, satisfaction, and orgasm may have been due to better PFM function after SESdS. In addition, proper functioning of the PFM is important for a woman's pleasure during vaginal intercourse, and for the strength of grip felt by her partner. 


\section{Obstetrics \& Gynecology Science}

Vol. 63, No. 3, 2020

Therefore, the improvements in the partner-related domain of the PISQ may have been influenced by an increase in the strength of a woman's grip.

The present study had some limitations. We did not measure changes in hypertrophy or activation of the PFM using ultrasonography or electromyography. Therefore, further studies are needed to investigate the effects of 8 weeks of transcutaneous ES training on changes in hypertrophy and activation of the PFM, as well as on PFM function. Also, further studies with a larger sample size should be performed to further elucidate FSD caused by PFM dysfunction.

In conclusions, the present study demonstrated that SESdS can improve PFM function (strength, power, and endurance) and female sexual function. SESdS can be used to improve sexual function in women with SUI. These results could be used to inform guidelines for treating FSD caused by PFM dysfunction.

\section{Acknowledgements}

The authors received financial and administrative support from the Yonsei University Research Fund (grant numbers: 2018-51-0213 and 2019-51-0094).We would like to thank all of the participants for their time and commitment to the present study.

\section{Conflict of interest}

No potential conflict of interest relevant to this article was reported.

\section{Ethical approval}

The study was approved by the Institutional Review Board at Yonsei University (Wonju, Korea; No. 1041849-201904-BM050-01).

\section{Patient consent}

The study procedures were explained to all subjects in advance, written informed consent was obtained.

\section{References}

1. Coyne KS, Zhou Z, Thompson C, Versi E. The impact on health-related quality of life of stress, urge and mixed urinary incontinence. BJU Int 2003;92:731-5.

2. Handa VL, Zyczynski HM, Burgio KL, Fitzgerald MP, Borello-France $D$, Janz NK, et al. The impact of fecal and urinary incontinence on quality of life 6 months after childbirth. Am J Obstet Gynecol 2007;197:636.e1-6.

3. Yip SK, Chan A, Pang S, Leung P, Tang C, Shek D, et al. The impact of urodynamic stress incontinence and detrusor overactivity on marital relationship and sexual function. Am J Obstet Gynecol 2003;188:1244-8.

4. Barber MD, Visco AG, Wyman JF, Fantl JA, Bump RC; Continence Program for Women Research Group. Sexual function in women with urinary incontinence and pelvic organ prolapse. Obstet Gynecol 2002;99:281-9.

5. Rogers GR, Villarreal A, Kammerer-Doak D, Qualls C. Sexual function in women with and without urinary incontinence and/or pelvic organ prolapse. Int Urogynecol J Pelvic Floor Dysfunct 2001;12:361-5.

6. Graber B, Kline-Graber G. Female orgasm: role of pubococcygeus muscle. J Clin Psychiatry 1979;40:348-51.

7. Castro RA, Arruda RM, Zanetti MR, Santos PD, Sartori MG, Girão MJ. Single-blind, randomized, controlled trial of pelvic floor muscle training, electrical stimulation, vaginal cones, and no active treatment in the management of stress urinary incontinence. Clinics (Sao Paulo) 2008;63:465-72.

8. Zahariou AG, Karamouti MV, Papaioannou PD. Pelvic floor muscle training improves sexual function of women with stress urinary incontinence. Int Urogynecol J Pelvic Floor Dysfunct 2008;19:401-6.

9. Beji NK, Yalcin O, Erkan HA. The effect of pelvic floor training on sexual function of treated patients. Int Urogynecol J Pelvic Floor Dysfunct 2003;14:234-8.

10. Rivalta M, Sighinolfi MC, Micali S, De Stefani S, Bianchi G. Sexual function and quality of life in women with urinary incontinence treated by a complete pelvic floor rehabilitation program (biofeedback, functional electrical stimulation, pelvic floor muscles exercises, and vaginal cones). J Sex Med 2010;7:1200-8.

11. Bø K, Talseth T, Vinsnes A. Randomized controlled trial on the effect of pelvic floor muscle training on quality of life and sexual problems in genuine stress incontinent 


\section{Obstetrics \& Gynecology Science}

Ui-Jae Hwang, et al. SESdS on PFM and sexual function

women. Acta Obstet Gynecol Scand 2000;79:598-603.

12. Lowenstein L, Gruenwald I, Gartman I, Vardi Y. Can stronger pelvic muscle floor improve sexual function? Int Urogynecol J Pelvic Floor Dysfunct 2010;21:553-6.

13. Martinez CS, Ferreira FV, Castro AA, Gomide LB. Women with greater pelvic floor muscle strength have better sexual function. Acta Obstet Gynecol Scand 2014;93:497-502.

14. Maher RM, Caulfield B. A novel externally applied neuromuscular stimulator for the treatment of stress urinary incontinence in women--a pilot study. Neuromodulation 2013;16:590-4.

15. Correia GN, Pereira VS, Hirakawa HS, Driusso P. Effects of surface and intravaginal electrical stimulation in the treatment of women with stress urinary incontinence: randomized controlled trial. Eur J Obstet Gynecol Reprod Biol 2014;173:113-8.

16. Green RJ, Laycock J. Objective methods for evaluation of interferential therapy in the treatment of incontinence. IEEE Trans Biomed Eng 1990;37:615-23.

17. Shamliyan TA, Kane RL, Wyman J, Wilt TJ. Systematic review: randomized, controlled trials of nonsurgical treatments for urinary incontinence in women. Ann Intern Med 2008;148:459-73.

18. Yokozuka M, Namima T, Nakagawa $H$, Ichie M, Handa Y. Effects and indications of sacral surface therapeutic electrical stimulation in refractory urinary incontinence. Clin Rehabil 2004;18:899-907.

19. Krauss DJ, Lilien OM. Transcutaneous electrical nerve stimulator for stress incontinence. J Urol 1981;125:790-3.

20. Fall M. Electrical pelvic floor stimulation for the control of detrusor instability. Neurourol Urodyn 1985;4:329-35.

21. Faul F, Erdfelder E, Buchner A, Lang AG. Statistical power analyses using $G^{*}$ Power 3.1: tests for correlation and regression analyses. Behav Res Methods 2009;41:1149-60.

22. Yoo EH, Suh JH, Lee SK. Translation and linguistic validation of Korean version of short form of PFDI (pelvic floor distress inventory) and PISQ (pelvic organ prolapse and incontinence sexual questionnaire). Proceedings of the 94th Annual Congress of Korean Society of Obstetrics and Gynecology; 2008 Sep 26-27; Seoul, Korea. Seoul: Korean Society of Obstetrics and Gynecology; 2008. p.184.

23. Rogers RG, Kammerer-Doak D, Villarreal A, Coates K, Qualls C. A new instrument to measure sexual function in women with urinary incontinence or pelvic organ prolapse. Am J Obstet Gynecol 2001;184:552-8.

24. Jha S, Walters SJ, Bortolami O, Dixon S, Alshreef A. Impact of pelvic floor muscle training on sexual function of women with urinary incontinence and a comparison of electrical stimulation versus standard treatment (IPSU trial): a randomised controlled trial. Physiotherapy 2018;104:91-7.

25. Bø K, Kvarstein B, Hagen R, Larsen S. Pelvic floor muscle exercise for the treatment of female stress urinary incontinence: I. Reliability of vaginal pressure measurements of pelvic floor muscle strength. Neurourol Urodyn 1990;9:471-7.

26. Tennfjord MK, Engh ME, Bø K. An intra- and interrater reliability and agreement study of vaginal resting pressure, pelvic floor muscle strength, and muscular endurance using a manometer. Int Urogynecol J Pelvic Floor Dysfunct 2017;28:1507-14.

27. Barbat-Artigas S, Rolland Y, Zamboni M, AubertinLeheudre M. How to assess functional status: a new muscle quality index. J Nutr Health Aging 2012;16:67-77.

28. Sacomori C, Cardoso FL. Predictors of improvement in sexual function of women with urinary incontinence after treatment with pelvic floor exercises: a secondary analysis. J Sex Med 2015;12:746-55.

29. Sale D, Norman R. Testing strength and power. In: MacDougall JD, Wenger HA, Green HJ, editors. Physiological testing of the high-performance athlete. Champaign (IL): Human Kinetics: 1991. p.21-106.

30. Harman E. Strength and power: a definition of terms. Strength Condit J 1993;15:18-21.

31. Wilmore JH, Costill DL, Kenney WL. Physiology of sport and exercise. Champaign (IL): Human Kinetics; 1994.

32. Dumoulin C, Seaborne DE, Quirion-DeGirardi C, Sullivan SJ. Pelvic-floor rehabilitation, part 1: comparison of two surface electrode placements during stimulation of the pelvic-floor musculature in women who are continent using bipolar interferential currents. Phys Ther 1995;75:1067-74.

33. Terlikowski R, Dobrzycka B, Kinalski M, Kuryliszyn-Moskal A, Terlikowski SJ. Transvaginal electrical stimulation with surface-EMG biofeedback in managing stress urinary incontinence in women of premenopausal age: a doubleblind, placebo-controlled, randomized clinical trial. Int Urogynecol J Pelvic Floor Dysfunct 2013;24:1631-8. 


\section{Obstetrics \& Gynecology Science}

Vol. 63, No. 3, 2020

34. Schoenfeld BJ. The mechanisms of muscle hypertrophy and their application to resistance training. J Strength Cond Res 2010;24:2857-72.

35. Gregory CM, Bickel CS. Recruitment patterns in human skeletal muscle during electrical stimulation. Phys Ther 2005;85:358-64.

36. Aydın S, Arıoğlu Aydın Ç, Batmaz G, Dansuk R. Effect of vaginal electrical stimulation on female sexual functions: a randomized study. J Sex Med 2015;12:463-9.

37. Kinsey AC, Pomeroy WB, Martin CE, Gebhard PH. Sexual behavior in the human female. Bloomington (IN): Indiana University Press; 1998.

38. Serati M, Braga A, Di Dedda MC, Sorice P, Peano E,
Biroli A, et al. Benefit of pelvic floor muscle therapy in improving sexual function in women with stress urinary incontinence: a pretest-posttest intervention study. J Sex Marital Ther 2015;41:254-61.

39. Salonia A, Zanni G, Nappi RE, Briganti A, Dehò F, Fabbri $F$, et al. Sexual dysfunction is common in women with lower urinary tract symptoms and urinary incontinence: results of a cross-sectional study. Eur Urol 2004;45:642-8.

40. Giuseppe PG, Pace G, Vicentini C. Sexual function in women with urinary incontinence treated by pelvic floor transvaginal electrical stimulation. J Sex Med 2007;4:702-7. 